\title{
L'utilisation de fonction radiale de base à support compact pour accélérer le temps de décomposition BEMD
}

\author{
Mohammed Arrazaki ${ }^{1,}$ Tarek Zougari ${ }^{2}$ \\ Département Mathématiques et Informatique, Faculté des Sciences Tétouan, Université Abdelmalek \\ Essaâdi Maroc \\ ${ }^{2}$ Département Mathématiques et Informatique, Ecole Nationale des Sciences Appliquées de Tanger, Université \\ Abdelmalek Essaâdi Maroc
}

Résumé: Cet article présente une nouvelle méthode de la Décomposition Modale Empirique Bidimensionnelle (BEMD). Cette méthode est basée sur l'utilisation des fonctions radiales de base à support compact (CSRBF) comme fonction d'interpolation à la place des fonctions radiales de base globales. La comparaison des deux méthodes, donne une qualité visuelle du même ordre, tout en remarquant une amélioration au niveau du temps de calcul. Ce qui présente le critère le plus important dans les applications en temps réel. Il a été montré que la méthode qui basée sur une interpolation par CSRBF donne un gain du point de vue du temps d'exécution.

Mots-clés : BEMD, fonction CSRBF, fonction RBF.

\section{Introduction}

La Décomposition Modale Empirique Bidimensionnelle (BEMD, Bidimensional Empirical Mode Decomposition) a permis d'élaborer des nouvelles approches pour le traitement et l'analyse multi-échelle des images et son utilisation a conduit à des résultats de meilleures qualités que les techniques existantes de décomposition, telles que la transformé de Fourier et les Ondelettes, nous allons présenter l'algorithme correspondant à la BEMD pour une image $\mathrm{I}(\mathrm{m}, \mathrm{n})[1]$.

1. Initialisation : on $\operatorname{poser}_{0}(\mathrm{~m}, \mathrm{n})=\mathrm{I}(\mathrm{m}, \mathrm{n}) \quad \mathrm{k}=1$

2. Extraction de la Kème IMF, notée $d_{k}(m, n)$ (Début de processus de tamisage).

a) Initialisation : $\mathrm{h}_{0}(\mathrm{~m}, \mathrm{n})=\mathrm{r}_{\mathrm{k}-1}(\mathrm{~m}, \mathrm{n}) \quad \mathrm{j}=1$

b) Extraire les minima et les maxima locaux de $h_{j-1}(m, n)$

c) Interpoler les minima locaux et les maxima locaux pour construire les enveloppes $\operatorname{EnvMin}_{j-1}(m, n)$ et $\operatorname{EnvMax}_{j-1}(m, n)$

d) Calculer la moyenne des deux enveloppes :

$$
\mathrm{m}_{\mathrm{j}-1}(\mathrm{~m}, \mathrm{n})=\left(\operatorname{EnvMin}_{\mathrm{j}-1}(\mathrm{~m}, \mathrm{n})+\operatorname{EnvMax}_{\mathrm{j}-1}(\mathrm{~m}, \mathrm{n})\right) / 2
$$

e) Mettre à jour $h_{j}(m, n)=h_{j-1}(m, n)-m_{j-1}(m, n)$ avec $j=j+1$

f) Calculer le critère d'arrêt:

$$
\mathrm{SD}(\mathrm{j})=\frac{1}{\mathrm{~N}} \sum_{\mathrm{t}=0}^{\mathrm{T}}\left[\frac{\left(\mathrm{h}_{\mathrm{j}-1}(\mathrm{~m}, \mathrm{n})-\mathrm{h}_{\mathrm{j}}(\mathrm{m}, \mathrm{n})\right)^{2}}{\mathrm{~h}^{2}{ }_{\mathrm{j}-1}(\mathrm{~m}, \mathrm{n})+\varepsilon}\right]
$$

Où $\varepsilon$ est un terme (faible) éliminé d'éventuelles divisions par zéro.

g) Décision pour le processus de tamisage:

- Si le critère d'arrêt est satisfait: $\mathrm{SD}(\mathrm{j})<\mathrm{SD}_{\max }$

Alors $\quad d_{k}(m, n)=h_{j}(m, n)$

- Sinon, retourner en (b) avec $\mathrm{j}=\mathrm{j}+1$

3. Mettre à jour le résidu $r_{k}(m, n)=r_{k-1}(m, n)-d_{k}(m, n)$

4. Décision pour le processus de décomposition :

- $\mathrm{Si} \mathrm{r}_{\mathrm{k}}(\mathrm{m}, \mathrm{n})$ a encore au moins quatre extrema, retourner en (2) avec $\mathrm{k}=\mathrm{k}+1$

- Sinon, la décomposition est terminée $r_{k}(m, n)$ est le résidu $r(m, n)$ de cette décomposition.

Ainsi, on obtient une décomposition de l'image sous la forme :

$$
\mathrm{I}(\mathrm{m}, \mathrm{n})=\sum_{\mathrm{K}=1}^{\mathrm{P}} \mathrm{d}_{\mathrm{k}}(\mathrm{m}, \mathrm{n})+\mathrm{r}(\mathrm{m}, \mathrm{n}) \quad \mathrm{P} \in \mathrm{N}^{*}
$$

Une amélioration des différentes phases de décomposition rendrait à priori un meilleur temps de calcul et donnerait des IMF's de meilleur qualité. La netteté des images exige une technique performante pour l'extraction des extrema, l'élaboration des méthodes d'interpolation à base d'autres fonctions d'interpolation et 
un critère d'arrêt adéquat et adaptatif à la nature de l'image permettant l'extraction de toute l'information utile et ainsi la réduction du temps de calcul.

Dans le processus de la décomposition, l'interpolation constitue une problématique pour l'estimation des surfaces formées par les extrema afin d'extraire les différentes IMF's. En effet, la construction des deux enveloppes inferieur et supérieur est faite par interpolation respectivement des maxima et minima. La dispersion de ces extrema entraine un problème du choix de la fonction d'interpolation.

Le reste de cet article est organisé comme suit: la section 2 donne un aperçu sur les fonctions radiales de base globale et les fonctions radiales de base à support compact. La section 3 décrit la complexité de l'algorithme. Ensuite, le papier continue à section 4, qui contient les résultats expérimentaux. Enfin, la section 5 pour la conclusion.

\subsection{Principe}

\section{Fonction d'interpolation Radiale de Base}

L'étape d'interpolation dans la procédure de BEMD joue un rôle décisif et qui a une incidence remarquable sur le résultat de la décomposition. En effet, l'extraction des IMF's repose essentiellement sur l'interpolation des points extrema pour créer les deux enveloppes : enveloppe inferieure et enveloppe supérieure. Plusieurs méthodes d'interpolation ont été proposées. Une étude comparative des méthodes d'interpolation pour le processus de tamisage a été présentée par [1]. Il en résulte que l'interpolation par les fonctions radiales de base Multi-Quadratique (MQ) et Thin-Plate-Spline (TPS) restent les plus adaptées.

\subsection{Les fonctions radiales de base}

Une des simples manières d'approcher une fonction $f$ connue en $N$ points distincts $X=\left\{x_{1}, \ldots \ldots, x_{N}\right\}$ est de choisir une fonction $\phi(x): \mathbb{R}^{d} \times \mathbb{R}^{d} \rightarrow \mathbb{R}$ et de formuler l'interpolant sous la forme :

tel que

$$
S_{f, X}(x)=\sum_{j=1}^{N} \alpha_{j} \phi\left(x, x_{j}\right)
$$

$$
S_{f, X}\left(x_{j}\right)=f\left(x_{j}\right) \forall j=1, ., N
$$

Autrement, on doit chercher le vecteur $\alpha=\left(\alpha_{i}\right)_{i=1 \ldots N}$ solution du système:

$$
\left(\begin{array}{ccc}
\phi\left(x_{1}, x_{1}\right) & \cdots & \phi\left(x_{1}, x_{N}\right) \\
\vdots & \ddots & \vdots \\
\phi\left(x_{N}, x_{1}\right) & \cdots & \phi\left(x_{N}, x_{N}\right)
\end{array}\right)\left(\begin{array}{c}
\alpha_{1} \\
\\
\alpha_{N}
\end{array}\right)=\left(\begin{array}{c}
f\left(x_{1}\right) \\
\\
f\left(x_{N}\right)
\end{array}\right)
$$

Le système (1) peut s'écrire sous la forme matricielle suivante

$$
A_{\phi, X} \alpha=F
$$

Avec $A_{\phi, X}=\left(\phi\left(x_{i}, x_{j}\right)\right)_{i, j \in[1, N]^{2}}$ une matrice de dimension $N \times N$ et $F=\left(f_{i}\right)_{i=1 \ldots N .}$ est le second membre déterminé à partir de la fonction $f$ et de l'ensemble des points distincts $\left\{x_{1}, \ldots \ldots, x_{N}\right\}$ données, Pour un choix donné de $\phi$, on peut déterminer le vecteur $\alpha$ dans le cas ou la matrice est inversible [5].

Mais le problème qui se pose est comment peut-on choisir la fonction $\phi$ pour que la matrice

$$
A_{\phi, X}=\left(\phi\left(x_{1}, x_{1}\right)\right)_{i, j \in[1, N]^{2}}
$$

soit inversible pour cet échantillon de points distincts?

Pour le système (1) admet une solution, on doit choisir la fonction $\phi$ définit positive ou complètement monotone, il existe plusieurs fonctions qui peuvent être utilisées comme fonction de base pour les fonctions radiales de base [1]. Dans ce cadre, nous allons étudie quelques fonctions radiales de base pour interpoler les extrema dispersées, qui sont extraites dans le processus de tamisage.

\section{Définition 1}

Une fonction $\phi: \mathbb{R}^{d} \rightarrow \mathbb{R}$ est dite fonction radiale de base s'il existe une fonction

telle que: $\quad \varphi(x)=\phi(r)$ avec $r=\|x\|_{d}$

$$
\varphi:[0,+\infty[\rightarrow \mathbb{R}
$$

où $\|.\|_{d}$ est la norme usuelle de $\mathbb{R}^{d}$.

On va présenter sous-dessous quelques fonctions à base radial

Exemples 1

$$
\begin{array}{cc}
\phi(\mathrm{x})=\mathrm{e}^{-\mathrm{x}} & \text { Gaussienne (GA) } \\
\phi(\mathrm{x})=\mathrm{x} \log (\mathrm{x}) & \text { Logarithme } \\
\phi(\mathrm{x})=\mathrm{x} & \text { Linéaire } \\
\phi(\mathrm{x})=\sqrt{\mathrm{x}^{2}+1} & \text { Multi-Quadratique (MQ) } \\
\phi(\mathrm{x})=\mathrm{x}^{2} \log (\mathrm{x}) & \text { Thin-Plate-Spline (TPS) }
\end{array}
$$


Les fonctions radiales de base $\boldsymbol{G A}$ et $\boldsymbol{M Q}$ sont des fonctions définies positives. Donc la matrice du système (1) engendrée par ces fonctions est inversible et la solution est unique pour ce cas. Mais certaines fonctions radiales de base telles que les fonctions $\boldsymbol{T P S}$ ne sont pas définie positive ni complètement monotone. Pour remédier à ce problème, il faut ajouter des restrictions à la matrice d'interpolation du système [5]. Il faut introduire d'autres conditions pour que la matrice soit inversible afin que le système admette une solution unique.

\section{Définition 2}

Soit $f$ une fonction définie de $\mathbb{R}^{d}$ à valeurs dans $\mathbb{R}$. Soit $f$ a des valeurs finis d'un nombre fini de points $X=\left\{x_{1}, \ldots \ldots, x_{N}\right\}$ distincts

L'interpolant RBF augmenté de $f$ revient à écrire l'interpolant de $f$ sous la forme :

avec la condition:

$$
S_{f, X}(x)=\sum_{i=1}^{N} \alpha_{i} \varphi\left(x-x_{i}\right)+\sum_{j=1}^{m} \mu_{i} p_{i}(x) \quad x \in \mathbb{R}^{d}
$$

$$
\sum_{j=1}^{m} \alpha_{i} p_{k}\left(x_{i}\right)=0 \quad k=1 \ldots m
$$

où $\left\{\mathrm{p}_{1}, \ldots \ldots, \mathrm{p}_{\mathrm{m}}\right\}$ est la base de $\mathrm{P}_{\mathrm{m}-1}$ l'espace vectoriel des polynômes de degré inférieur ou égal à $\mathrm{m}-1$ définis de $\mathbb{R}^{d}$ à valeurs dans $\mathbb{R}$ et $m$ un entier naturel.

\subsection{Les fonctions radiales de base à support compact}

Une famille des CSRBFs a été introduite par $\mathrm{Wu}$, puis plus par Wendland vers le milieu des années 1990 [5]. Généralement une fonction radiale de base à support compact est donnée par l'expression $\phi_{\mathrm{l}, \mathrm{k}}(\mathrm{r})=(1-\mathrm{r})_{+}^{\mathrm{n}} \mathrm{p}(\mathrm{r}) \quad$ pour $k \geq 1$

avec les conditions suivantes

$$
(1-r)_{+}^{\mathrm{n}}=\left\{\begin{array}{cc}
(1-r)^{\mathrm{n}} \text { si } 0 \leq \mathrm{r}<1 \\
0 & \text { sir } \geq 1
\end{array}\right.
$$

où $p(r)$ est l'un des polynômes prescrits par Wu ou Wendland, Les indices $l$ et $2 k$ représentent respectivement la dimension et la souplesse de la fonction [5].

Quelques fonctions de Wu et Wendland

$$
\begin{gathered}
\phi_{2,0}(r)=(1-r)_{+}^{5}\left(1+5 r+9 r^{2}+5 r^{3}+r^{4}\right) \\
\phi_{2,1}(r)=(1-r)_{+}^{4}\left(4+16 r+12 r^{2}+3 r^{3}\right) \\
\phi_{3,0}(r)=(1-r)_{+}^{3} \\
\phi_{3,1}(r)=(1-r)_{+}^{4}(1+4 r)
\end{gathered}
$$

\section{ALGORITHME}

L'utilisation des fonctions radiales de base à support compact, offre des avantages dans les trois phases de la reconstruction de la surface [4].

\subsection{Construction du système}

\subsubsection{La fonction radiale de base (TPS)}

Une partie importante du coût de calcul des surfaces implicites est le coût nécessaire pour construire la matrice d'interpolation, pour les fonctions radiales de base cela signifie que la matrice est entièrement non nulle sauf le long de la diagonale, ce qui nécessite le calcul de l'ensemble des distances entre les points dans l'ensemble $\left\{x_{i}\right\}_{i \in[1, N]}$, la complexité de calcule est $O\left(N^{2}\right)$.

\subsubsection{Les fonctions radiales de base à support compact}

A cause des fonctions radiales de base à support compact ont un support fini, on utilise l'arbre $K d$-tree (Bentley, 1975) [3], pour déterminer l'ensemble de tous les points dans un support d'un point particulier. Un Kd-tree est une structure de donnée pour la division spatiale de l'espace en $K$ dimensions. Un $K d$-tree est implanté comme un arbre binaire de recherche multi-niveaux.Afin d'avoir un arbre équilibré, la construction de l'arbre fait appel à une étape de tri linéaire pour trouver le point médian. Un tri linéaire en $O(N)$ donne une construction de l'arbre en $O(N \log N)$. Une fois l'arbre construit, on peut déterminer tous les points dans un support donné en $O(N)[2]$. 


\subsection{Résolution du système}

\subsubsection{La fonction radiale de base (TPS)}

On utilise la factorisation $L U$ de matrice symétrique définit positive pour résoudre le système linéaire, cela signifie qu'il est possible de le résoudre en $O\left(N^{3}\right)$

\subsubsection{Les fonctions radiales de base à support compact}

Pour résoudre le système linéaire, on utilise la factorisation $L U$ d'une matrice creuse, pour trouver la solution du système d'équations. La complexité de calcul d'un tel système dépend de la quantité de matrice "remplir ou non par les zéros" qui se produit au cours de la solution, mais certains auteurs ont signalé un comportement dans l'ordre $O\left(N^{1.2}\right)$ à $O\left(N^{1.5}\right)$ [4].

\subsection{L'évaluation de la fonction d'interpolation}

\subsubsection{La fonction radiale de base (TPS)}

Pour compléter l'algorithme il ne suffit pas de résoudre le système, il est nécessaire d'évaluer la fonction d'interpolation $S$ en nombreux points afin d'extraire la surface reconstruite, et chaque évaluation de fonction d'interpolation est nécessite $O(N)$.

\subsubsection{Les fonctions radiales de base à support compact}

Nous pouvons exploiter la localité spatiale des fonctions radiales de base à support compact lors de l'évaluation de la fonction $S$ d'interpolation. En utilisant de nouveau un arbre kd-tree pour organiser les points dans l'espace, chaque évaluation de la fonction d'interpolation ne nécessite que $O(\log (N))$ pour les déterminer.

\section{Résultats}

Nous allons présenter la décomposition BEMD d'une image texturée $113 * 113$ de l'extension $J P E G$, les simulations ont été effectuées avec le logiciel Matlab 7.12.0 (R20011a) sur un ordinateur Intel(R) Core(TM) 2 Duo avec une vitesse du microprocesseur égale à $2 \mathrm{GHz}$ et une RAM de $3 \mathrm{~Gb}$.

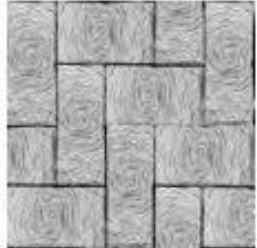

A

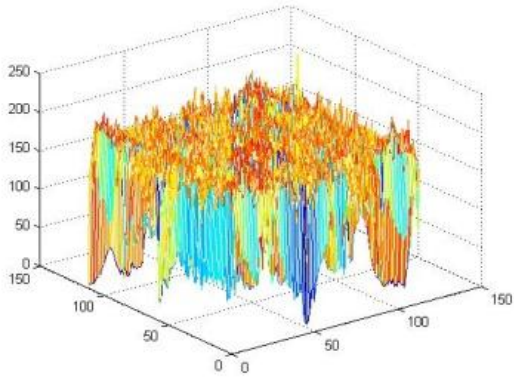

B

Fig1.A) image originale, B) Maillage en 3 dimensions de l'image original.

\section{La BEMD en utilisant la fonction RBF (TPS)}

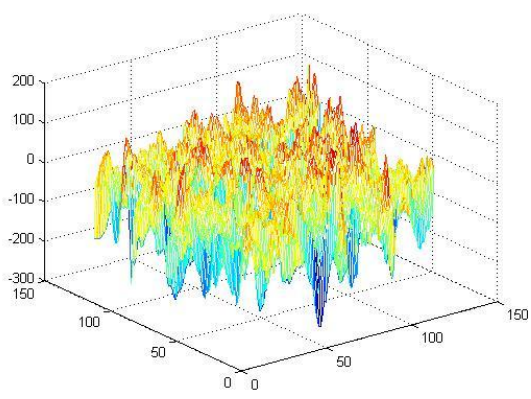

$1^{\grave{e} r e} I M F$

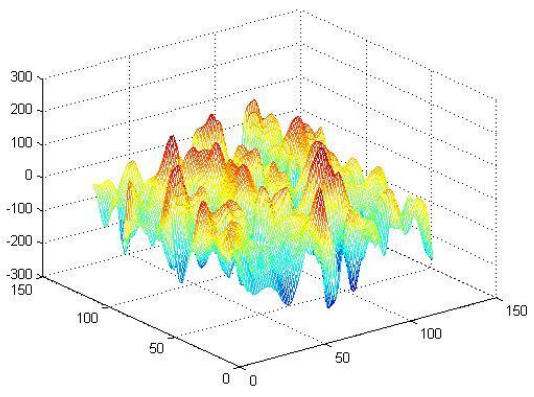

$2^{\text {ème }} I M F$ 


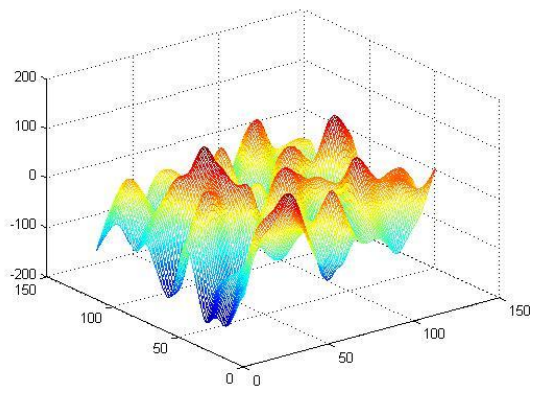

$3^{\text {ère }} I M F$

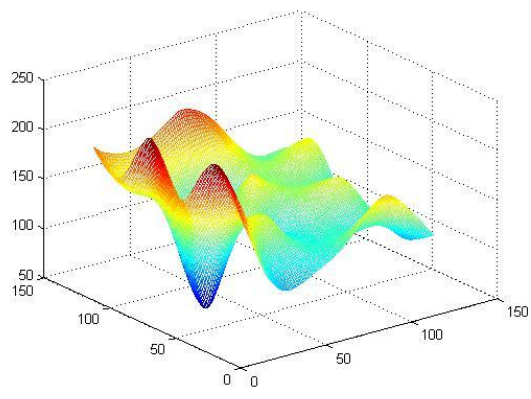

résidu

Fig2. La décomposition par la BEMD en utilisant la fonction Thin-Plate-Spline $\operatorname{TPS} \phi(\mathrm{x})=\mathrm{x}^{2} \log (\mathrm{x})$ comme la fonction d'interpolation (avec un temps de calcul $323.788225 \mathrm{~s}$ ).

\section{La BEMD en utilisant la fonction CSRBF}

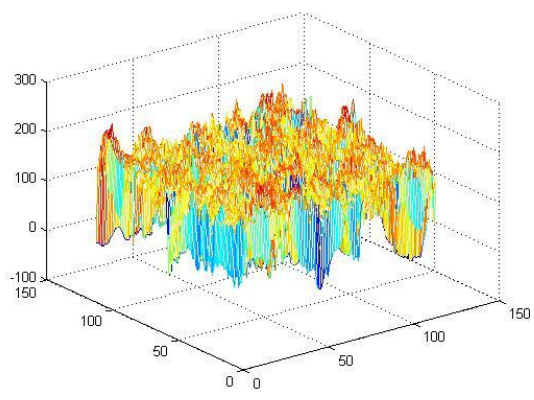

$1^{\text {ère }} I M F$

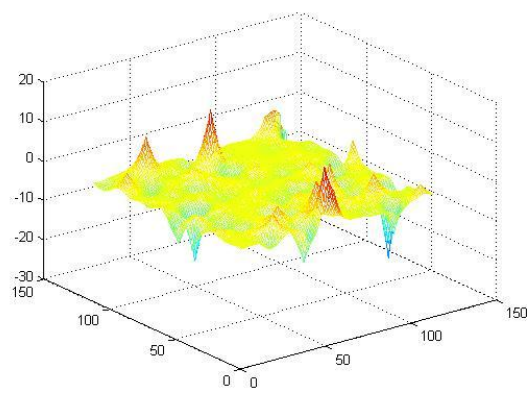

$3^{\text {ème }} I M F$

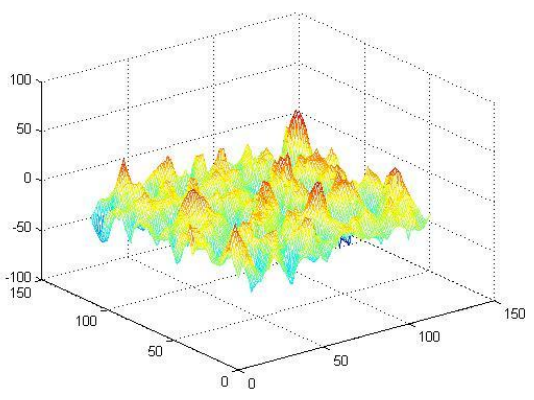

$2^{\text {ème }} I M F$

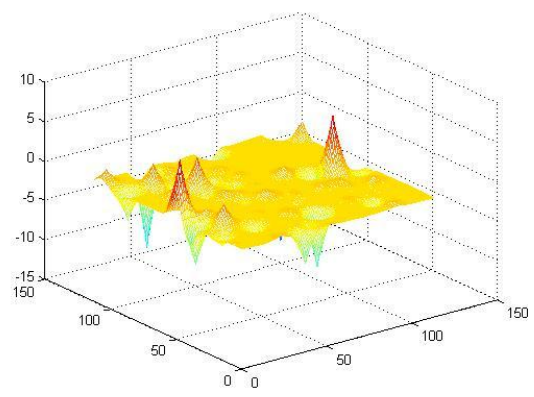

résidu

Fig3. La décomposition par la BEMD en utilisant la fonction CSRBF $\phi_{2,0}(r)=(1-r)_{+}^{2}$ comme fonction d'interpolation (avec un temps de calcul $54.459903 \mathrm{~s}$ ).

\section{Conclusion}

Le paragraphe précédent présente les résultats de la BEMD en utilisant l'interpolation par fonction radiale de base (Thin-Plate-Spline) et la fonction radiale de base à support compact (fonction de Wendland). Le rayon du support utilisé a été choisi constant.

Les résultats des deux méthodes sont qualitativement presque identiques, puisque les deux résidus ne contiennent aucune information sur l'image originale, mais le temps nécessaire montre la différence entre ces deux méthodes, puisque la BEMD avec l'interpolant RBF (Thin-Plate-Spline) prend $323.788225 \mathrm{~s}$, par contre l'interpolant CSRBF (Windland) ne dépasse pas $54.459903 \mathrm{~s}$, ce qui va être plus intéressant quand utilisant les images de grand dimension. Ces résultats sont conformes aux résultats de complexité de calcul de la BEMD au niveau de la construction du système, résolution du système et L'évaluation de la fonction d'interpolation. D'après les résultats obtenus, l'utilisation de l'interpolant CSRBF (Windland) permet de faire une amélioration en temps de calcul. 


\section{Références}

[1] A. Sabri, Décomposition Multimodale Empirique Bidimensionnelle : Améliorations et Applications, doctorat national UFR : Automatique et analyse des systèmes, le 13 juin 2009.

[2] M. De Berg, O. Cheong, and M. van Kreveld. Computational Geometry: Algorithms and Applications. Springer, 2008.

[3] J. L. Bentley. Multidimensional binary search trees used for associative searching. CACM, 18(9):509-517, 1975.

[4] B S. Morse, T S. Yoo, P Rheingans, D T. Chen, K. R. Subramanian. Interpolating Implicit Surfaces From Scattered Surface DataUsing Compactly Supported Radial Basis Function. Department of Computer Science, Brigham Young University, 2005.

[5] Gregory E. Fasshauer, Meshfree Approximation Methods with Matlab vol.6, Illinois Institute of Technology, USA 2007. 